\title{
The Relationship Between Photospheric Magnetic Field Evolution and Major Flares
}

\author{
Y. Zhang, J.H. Liu \\ and H.Q. Zhang \\ ${ }^{1}$ National Astronomical Observatories, Chinese Academy of Sciences, Beijing 100012, China \\ email: zhy@sun10.bao.ac.cn
}

\begin{abstract}
On 28 Oct 2003, one of the biggest flares (4B/X17.2) seen in recent years occurred in Active Region (AR) NOAA 10486 associated with a violent halo coronal mass ejection. It was a complex $\beta \gamma \delta$ region. After studying the evolution of the AR and the phenomena of this powerful flare, we obtained the following result. (1) Highly sheared transverse field was formed gradually on both sides of the neutral line by squeeze during the AR development; (2) Rotations of penumbra of main polarities were discerned, and the average horizontal velocities was as large as $0.55 \mathrm{~km} / \mathrm{s}$; (3) The spiral transverse field of main positive polarity was diffused after the large flare; (4) Some magnetic features submerged or emerged in the vicinity of the flare onset point. The emergence of this rotational and complex magnetic topology implies a transport of magnetic energy and complexity from the low atmosphere to the corona. Moreover, the rapidly submergence (emergence) and movements of the small magnetic features which represent the enhancement (cancellation) and squeeze of the magnetic field play a key role in the onset of the flare.
\end{abstract}

Keywords. sun: flare — sun: magnetic field—sun: activity

\section{Observation}

For our current study, the data are from the following source: 1. complex sets of vector magnetograms from Huairou Solar Observing Station (HSOS) of the National Astronomical Observatories of China; 2. white light and EUV images from Transition Region and Coronal Explorer (TRACE) satellite.

\section{Result}

\subsection{Long Term Evolution}

The AR NOAA 10486 was a complex $\beta \gamma \delta$ configuration groups during its passage across the solar disk from Oct 22 to Nov 2 (Zhang, et al. 2003). The most important evolutionary mechanisms in this region are: 1 . gradually formed highly sheared transverse field and longitudinal grad on both sides of the neutral line by squeeze during the AR development (Fig. 1); 2. different rotational pattern of sunspots (Fig. 2). The spiral magnetic structure which covered the whole P2 on Oct 28 diffused after the flare. The penumbra shared by P1 and P2 had a counterclockwise rotation pattern, while N4 and P3 had clockwise rotation pattern. N3 was squeezed by the different rotational polarities on both sides and developed as slender negative region.

\subsection{Onset of the Flare}

We present the time sequence of TRACE $171 \AA$ images in Fig. 3. Seen from $171 \AA$ movie, the AR showed various manifestations of activation before the powerful flare. It slowly 


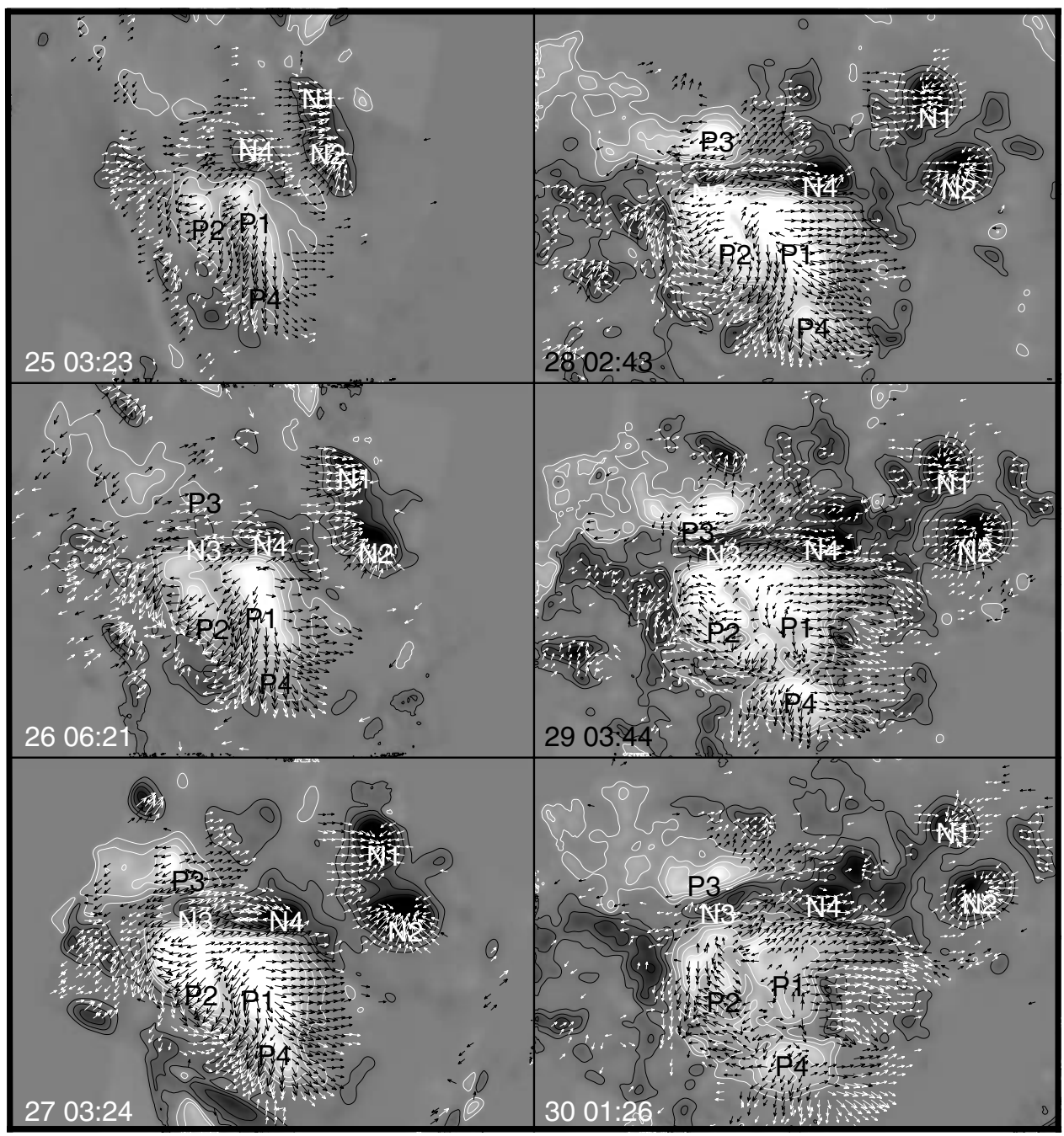

Figure 1. HSOS vector magnetograms in AR 10486. The longitudinal component of the magnetic field is presented by gray-scale patches and isogauss contours with levels of $\pm 200,500,900$ and $1400 \mathrm{G}$. White patches represent positive polarity fields and black patches represent negative fields. The transverse components of field is shown with short lines, with lengths proportional to the relative field strength. The size of images is $4.75^{\prime} \times 3.34^{\prime}$.

rose, meanwhile brightening appeared at part of the filament. Then it split into threads and each thread rose and fluctuated. Some filament materials ejected from 09:50 UT. At 10:17 UT part of the filament became brighten and rose rapidly, finial it erupted partially. At 11:01 UT, the filament erupted and a powerful two-ribbon flare developed rapidly.

\subsection{Rapid Change Association with Flare}

Disappearing sunspots which were located in the vicinity of highly sheared neutral line are outlined by windows 1 in Fig. 4 . These spots were merged and canceled with nearby opposite polarity P3 and totally disappeared around 08:00 UT. After the flare, shear angle here was declined from $60^{\circ}$ to $23^{\circ}$. Arrows 1 and 2 mark the emerging sunspots. Arrow 1 represents a penumbral feature which continuously emerged and developed as a little spot around 06:00 UT. The spot indicated by arrow 2 was already developed as 


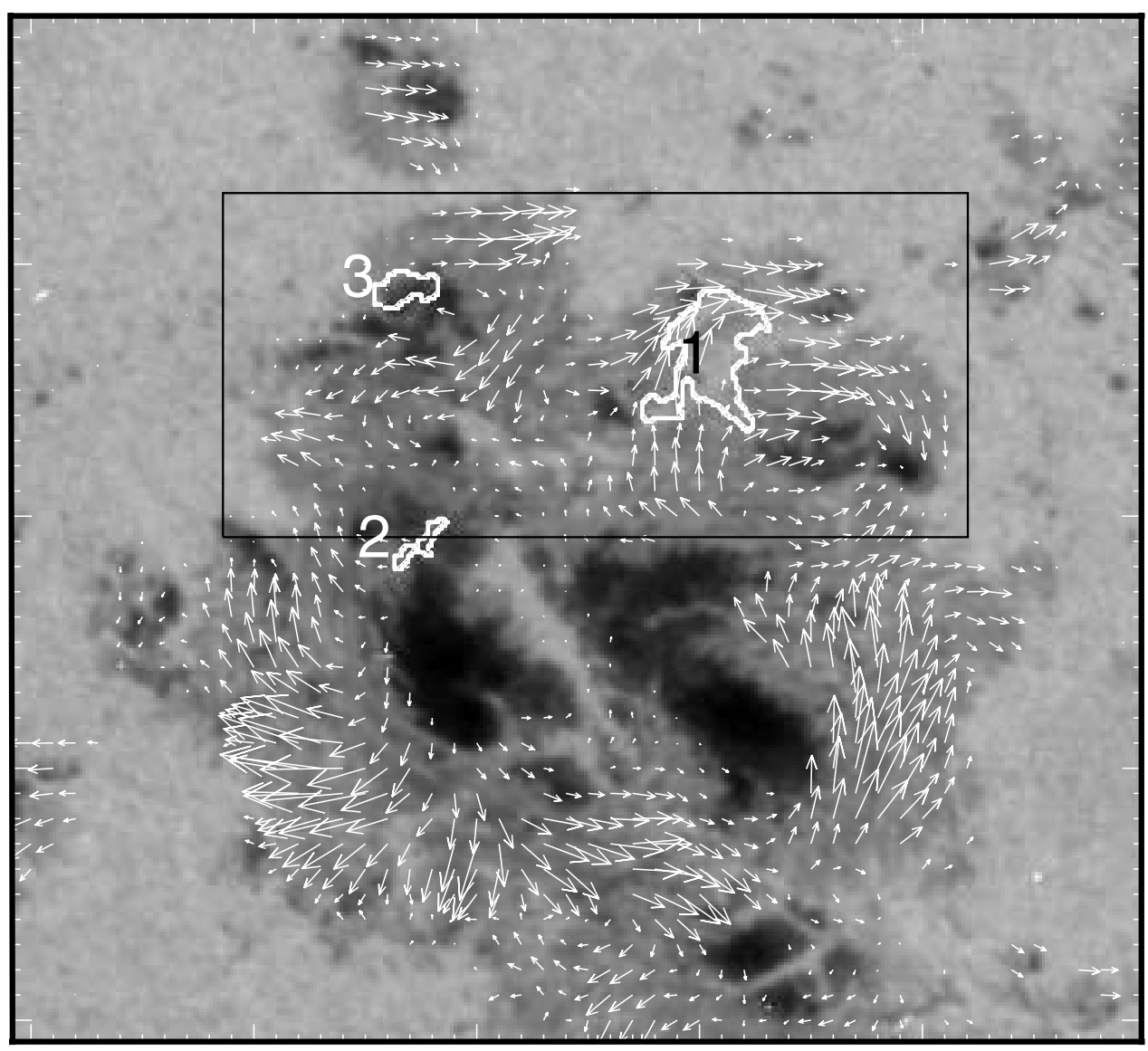

Figure 2. The average horizontal velocities is shown with arrows, and the length is proportional to the relative value of the velocity. The thick contours are the site of the flare onset. See details in the text.

a little spot on Oct 28 01:44 UT as shown in Fig. 4. It moved with spot 1 in the same direction rapidly. And before the powerful flare, they were already on the north of N4.

\section{Acknowledgements}

The authors would like to thank the staff in Huairou group for their comments and discuss. We are grateful to all members of TRACE teams for providing the wonderful data.

\section{References}

Zhang, H.Q., Bao, X.M., Zhang, Y., Liu, J.H., Bao, S.D., Deng, Y.Y., et al. 2003, ChJAA, 3, 491. 


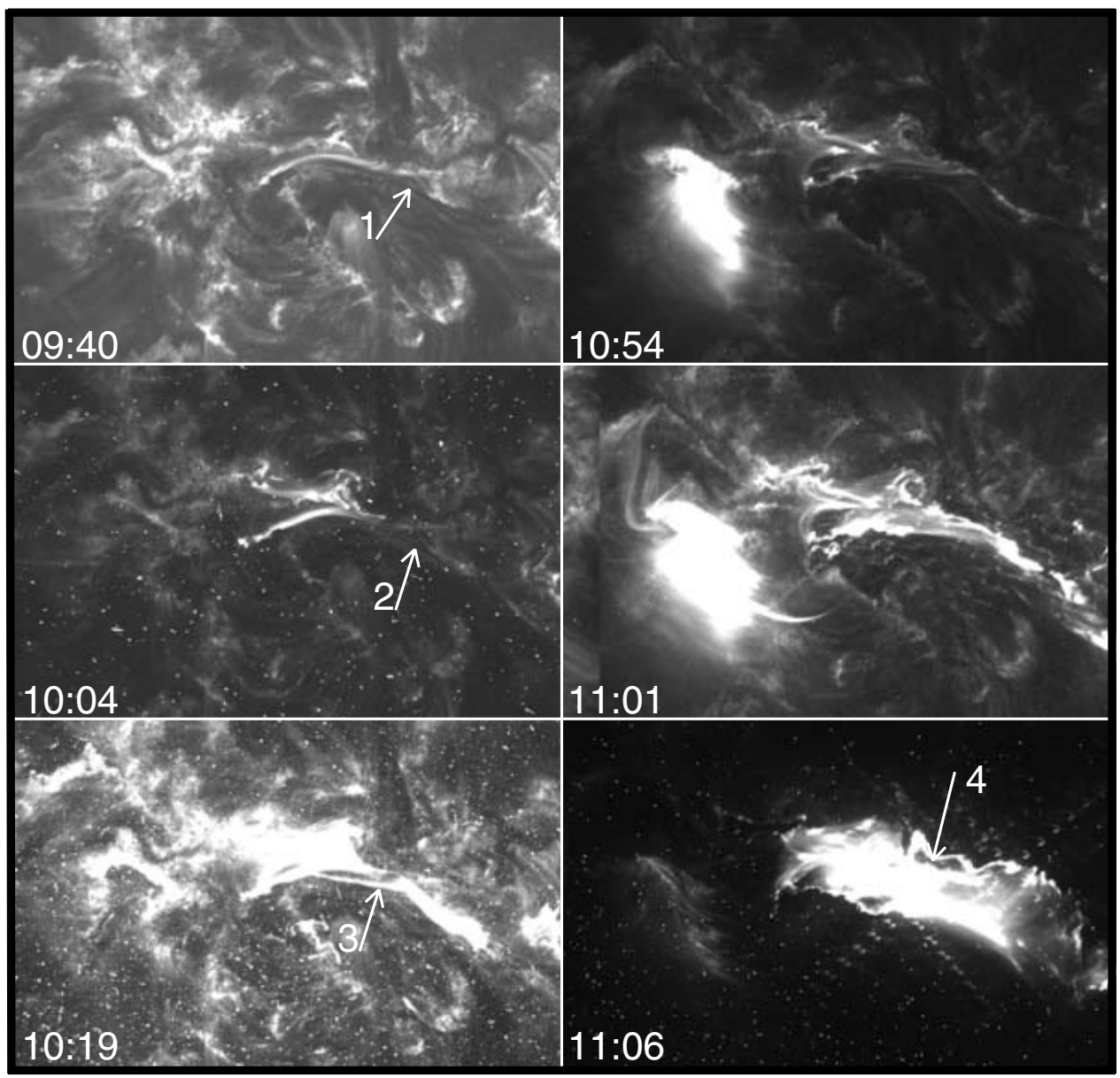

Figure 3. A series of $171 \AA$ images obtained by TRACE. Arrows indicate: (1) the filament; (2) the ascend of filament; (3) brightening of the filament; (4) the post flare loops;

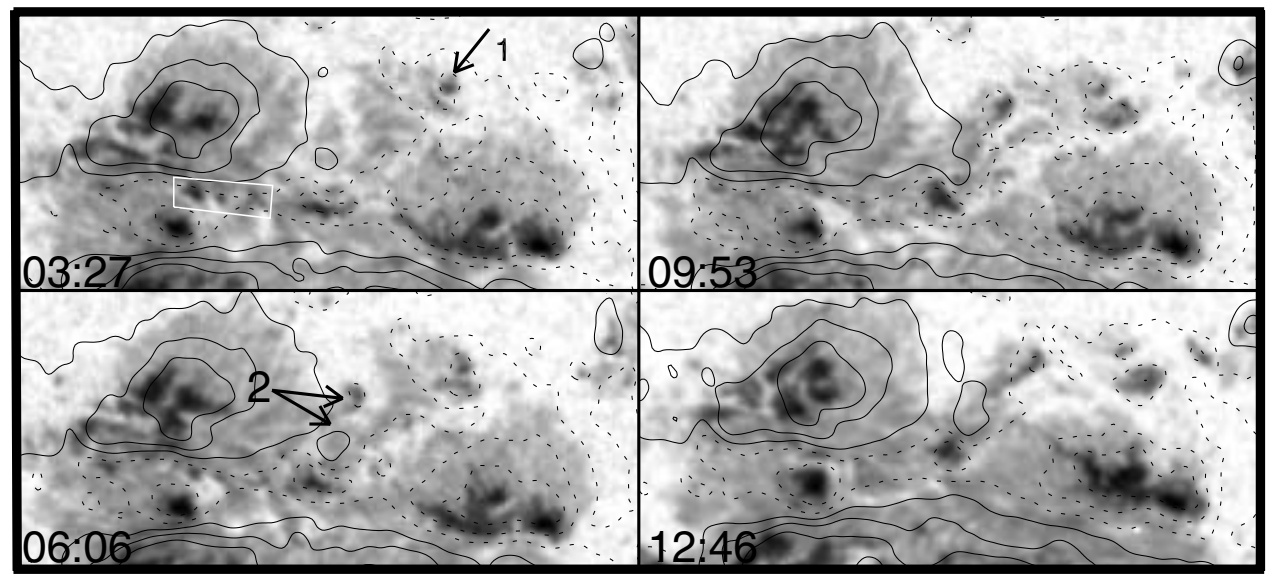

Figure 4. The rapid evolution of the sunspots. The window and arrows are described in the text. 